\author{
Klaus Peter Hufer \\ Universität Duisburg Essen
}

\title{
Was hält die Gesellschaft noch zusammen? Politische Bildung in einer entgrenzten Zeit
}

\author{
Co jeszcze spaja społeczeństwo? Edukacja obywatelska \\ w czasie bez czasu
}

Streszczenie. Niniejszy artykuł opisuje sytuację edukacji obywatelskiej dorosłych w Niemczech oraz wyzwania, przed którymi współcześnie stoi. Jesteśmy świadkami rozpadania się społeczeństwa i przewagi anomii, obojętności, egoizmu nad dążeniami do zachowania spójności. Co może wnieść edukacja obywatelska do tego, aby społeczeństwo stanowiło wspólnotę? W odpowiedzi na to pytanie w artykule są prezentowane cztery palące kwestie:

1. Sytuacja społeczna: żyjemy w niejasnych, sprzecznych czasach; nie ma analiz społecznych, które pozwalają spojrzeć na przyszłość z optymizmem; socjolodzy i politolodzy konstatują dezorganizację i wycofanie; Jacques de Saint Victor (2015) opisuje fenomen „antypolityki" w Europie; antyinstytucjonalny populizm i nienawiść wobec kast: polityków, przedsiębiorców, dziennikarzy, których nazywa się „zdrajcami” i „kłamcami”; „zatroskani obywatele" protestują w sieci i na ulicy, ale miejsce niezależności zajęła głupota, zamiast kultury mamy wulgarność, zamiast tolerancji - brak wyrozumiałości, zamiast otwartości - zamknięcie; spadło zaufanie wobec instytucji społecznych: na ostatnim miejscu są partie (23\% zaufania społecznego), nieco wyżej kościół (31,1\% zaufania społecznego), komercyjne radio i media społeczne (35\%/36\% zaufania społecznego); największym zaufaniem cieszy się policja (65,5\%) oraz Trybunał Konstytucyjny (63,5\%) (Decker, Kies, Brähler 2016, s. 60).

2. Dyskusja na temat rozumienia edukacji i polityki: pojęcia te coraz trudniej zdefiniować w pluralistycznym, zindywidualizowanym, podzielonym społeczeństwie świata; za kluczowe pojęcia edukacji uznaje się niezależność (Aufklärung) oraz odpowiedzialność (Mündigkeit); w edukacji politycznej chodzi o zrozumienie, że nie ma lepszego systemu niż socjalna demokracja, wiedzę na temat demokratycznych zasad i sposobów podejmowania decyzji, zaangażowanie na rzecz zachowania i obrony praw człowieka oraz odrzucenie ekstremizmów, totalitaryzmów oraz dyskryminacji; „Demokracja to jedyny polityczny porządek społeczny, którego trzeba się uczyć - ciągle na nowo, codziennie, aż po starość" (Negt 2010, s. 174); edu- 
kacji politycznej nie można wyjąć z kontekstu: gdzie żyjemy? jakie są aktualne uwarunkowania polityczne?

3. Rozumienie profesji i zawodu przez tych, którzy pracują w instytucjach edukacji dorosłych: kadra edukacji dorosłych w Niemczech pracuje bardzo często w różnych miejscach po prostu w zamian za otrzymywane honorarium, rzadko na podstawie umowy o pracę w jednej instytucji - to przynosi różnorodne problemy związane z tożsamością zawodową; badania dotyczące nauczycieli zajmujących się edukacją polityczną pokazują, że nauczyciel w instytucjach edukacji dorosłych oraz w szkole to dwa zupełnie różne zawody. Mimo tego w przypadku nauczycieli osób dorosłych można zidentyfikować wspólne profesjonalne standardy, tj. kompetencje, których każdy potrzebuje, aby profesjonalnie działać w analizowanym obszarze. Christine Zeuner zaliczyła do nich kompetencje merytoryczne, społeczne i refleksyjność (2013, s. 85), które nie są wcale oczywiste i dlatego muszą być ciągle dyskutowane, pielęgnowane i strzeżone.

4. Aktualne polityczne problemy i społeczne tendencje oraz odpowiedzi, których może udzielić edukacja obywatelska: miejsca, gdzie panuje demokracja, są lekcjami edukacji obywatelskiej; w nowoczesnych społeczeństwie zanikają głębokie struktury poznawcze (Ligaturen), które umożliwiają ludziom znaleźć swoją drogę w świecie różnych opcji - edukacja obywatelska może dać wsparcie w tym zakresie; edukacja obywatelska może przyczynić się do budowania spójności społecznej jako część edukacji etycznej, kulturalnej i innych obszarów edukacji humanistycznej; instytucje edukacji obywatelskiej mogą wspierać odwrót od wyobcowania dzięki ofercie oświecenia rozumu. Według Bertolda Brechta „Myślenie należy do największych przyjemności ludzkiej rasy" (1968, s. 35) - programy tv, typu talk show, banalizują społeczne problemy - edukacja obywatelska troszczy się o budowanie poglądów opartych na argumentach; edukacja obywatelska może być kompasem w złożonym środowisku życia, wartościową i pouczającą odpowiedzią na wszechobecną konsumpcję; postępująca globalizacja potęguje poczucie wyobcowania - edukacja obywatelska podejmując zagadnienia stereotypów i uprzedzeń może przeciwdziałać temu; społeczeństwo obywatelskie czerpie siły witalne z różnorodności biografii, pochodzenia, kultur - edukacja obywatelska służy tym wartościom poprzez obronę praw człowieka i szerzenie tolerancji; internet stwarza możliwości szerokiej komunikacji, która służy demokracji, jednak internet wyzwolił także solipsyzm i dał upust skrajnościom; edukacja obywatelska dzięki obecności w sieci wspiera polityczną refleksję i kwestionuje antypolityczne resentymenty.

Słowa kluczowe: Niemcy; edukacja obywatelska dorosłych; społeczeństwo; globalizacja.

Summary. The following article describes the situation of political adult education in Germany and the challenges it is facing. The state of society is viewed as "unbounded". In light of this result, what can political adult education contribute to saving the cohesion of a society? In answering this question, four current foci will be described: 1 . The development of society, 2. The discussion about an understanding of education and politics, 3. The professional self-concept and the job image of those who work for institutions of political adult education, 4 . The contemporary political issues and social changes and the answers that political education may be able to give.

Key words: Germany, political adult education; society, globalization. 


\section{Der Blick auf die politische Erwachsenenbildung}

Wer politische Bildung organisiert, lehrt oder bildungspolitisch vertritt, hat selten richtig schöne Erfolgserlebnisse. Denn so recht weiß keiner, was aus den Angeboten, den Tagungen, Seminaren, Workshops, Vorträgen, den zur Verfügung gestellten Schriften folgt. Die Wirkung kann nicht eindeutig belegt werden. Sicher ist aber: politische Bildung ist eine stabile Säule der Demokratie. Ohne sie - das behaupte ich jetzt - wären die Probleme unserer Gesellschaft - und wir haben davon nicht wenige - noch größer, als sie bereits sind.

Da das noch von niemandem zwingend und empirisch eindeutig belegt ist, müssen sich politische Bildner/-innen mit kleinen Erfolgserlebnissen zufriedengeben. Ich hatte eines, als mir der Teilnehmer einer meiner Veranstaltungen Folgendes sagte: „Ich besuche gerne Veranstaltungen zu Politik und Gesellschaft, denn hier lerne ich interessante Meinungen kennen, mit denen ich mich auseinandersetzen kann. Und es geht nicht so trocken zu wie damals in meinem Sozialkundeunterricht in der Schule."

Im Folgenden konzentriere ich mich also auf die außerschulische politische Bildung, vor allem auf die politische Erwachsenenbildung. Aber die dargestellten Aspekte sind auch auf die politische Jugendbildung übertragbar.

Es ist eine alles andere als leichte Arbeit, die diejenigen, die hier tätig sind, übernommen haben, und viele Meriten sind auch nicht zu gewinnen: „Für die Bevölkerung, ja selbst für viele Erwachsenenbildungs- und Politikdidaktikstudierende ist politische Erwachsenenbildung oftmals eine Terra incognita. Um einen Vergleich mit klassischen Professionen zu bemühen: Trotz berechtigter Kritik gelten Ärzte nicht weniger als 'Götter in Weiß’, Priester als moralische Instanzen, Richter als geachtete Respektpersonen und Erwachsenenbildner? In der Öffentlichkeit werden sie kaum wahrgenommen, in der einschlägigen Literatur nicht selten unter DilettantismusVerdacht gestellt [...]. Es fordert sie Phantasie heraus, sich einen politischen Erwachsenenbildner als Roman-Helden, Spielfilm-Protagonisten oder Identifikationspunkt kindlicher Berufswünsche vorzustellen“" (Scheidig 2013, S. 10).

Immerhin hat dieses Manko dazu veranlasst, mit neueren Publikationen das höchst kreative Feld der außerschulischen politischen Jugendbildung und der politischen Erwachsenenbildung darzustellen und in seinen vielen Facetten auszuleuchten (z.B. Baltzter/Ristau/Schröder 2014; Hufer/ /Lange 2016; Hufer 2016).

Es ist schon einiges gelungen bei dem Bemühen, die außerschulische politische Bildung aus ihrem langjährigen Schattendasein herauszuholen. 
Das reicht aber noch nicht, sie muss weiter gefördert und ausgebaut werden. Dafür möchte ich werben.

Denn ich schaue mit Sorgen auf die Entwicklung unserer Gegenwart. Wir leben in entgrenzten Zeiten, in denen unsere Gesellschaft auseinanderdriftet. Aber es bedarf eines Zusammenhalts, sollen nicht Anomie, Gleichgültigkeit und Egoismus die bestimmenden Merkmale und Handlungen der hier lebenden Menschen sein. In der politischen Bildung dagegen wird in reflexiver Weise über ein verantwortungsethisches Handeln nachgedacht.

Jedoch ist ihr Feld nicht einfach bestellt, es gibt da einige Konflikte und Friktionen.

\section{Erster Brennpunkt: die Entwicklung der Gesellschaft}

Wir befinden uns in unklaren, widersprüchlich erlebten und sich darstellenden Zeiten. Eine klare Gesellschaftsanalyse, die hoffnungsvoll den Blick nach vorne zeigt, gibt es nicht mehr. Utopien sind verschwunden. Stattdessen konstatieren die Soziologen und Politologen Zerrüttung und Rückzug.

Der französische Politik-Professor Jacques de Saint Victor hat einen aktuellen Trend und die Menschen, die ihm hinterherlaufen, mit einem starken Begriff markiert: „Die Antipolitischen“ (Saint Victor 2015). In seinem gleichnamigen Buch beschreibt er ein europaweit wachsendes Phänomen, die „Antipolitik“. „Es bezeichnet eine Art moralischer Entrüstung und Rebellion vonseiten wachsender Randgruppen der Öffentlichkeit, die bestrebt sind, sich von der alten Politik zu befreien, vor allem durch die »Tugenden« des Netzes" (Saint Victor 2015, S. 10 i nast.). Als einen Grund dafür sieht Saint Victor ein „Jahrhundertübel“ der westlichen Welt, nämlich eine „Vertrauenskrise“ (Saint Victor 2015, S. 17). Eine absurde Situation ist entstanden: Einerseits ein „Kult des Privaten“ (Saint Victor 2015, S. 22), andererseits „steigert das Web 2.0 die Möglichkeit, »das Wort zu ergreifen«, ins Unermessliche" (Saint Victor 2015, S. 30).

Der einfache Klick im Internet erzeugt in der Tat ein nahezu omnipräsentes und -potentes Gefühl. Doch ist das eine qualitative Verbesserung der Demokratie? Der Autor verneint das. Er sieht ein wachsendes „Bündnis von Netz und Straße" (Saint Victor 2015, S. 33). Ein "antiinstitutioneller Populismus“" (Saint Victor 2015, S. 42) sei entstanden. Dessen Feind sei die „»Kaste«: die Kaste der Politiker, die Kaste der Unternehmer, die Kaste der Journalisten“ (Saint Victor 2015, S. 42). Wer denkt jetzt nicht an Pegida? Deren Protagonisten und Mitläufer organisieren sich per Internet, behaup- 
ten, sie seien "das Volk“ und pöbeln auf der Straße pauschalierend gegen "die“ Politik und „die“ Journalisten, deren Repräsentanten sie als „Volksverräter" oder „Lügenpresse“ bezeichnen.

Für uns politische Bildner/-innen ist dieses Phänomen verwirrend. Immer wieder haben wir ja Partizipation und Demokratisierung gewollt und in unseren Vorträgen, Kursen und Seminaren darauf hingezielt. Nun äußern sich millionenfach „besorgte Bürger“ im Netz und auf der Straße, aber statt Aufklärung ist Borniertheit, statt Kultur ist Vulgarität, statt Toleranz ist Unduldsamkeit statt Offenheit ist Enge entstanden. Und das verbreitet sich.

Immer schon hatte politische Bildung zwar dazu aufgerufen, die „Herrschenden“, das „Establishment“" kritisch zu hinterfragen. Jetzt aber wird pauschaler Hass skandiert gegen „Politiker, Wirtschaftsvertreter, Medienleute - sie alle gehören zum vermeintlichen Establishment derer "da oben " " (Nachtwey 2016, S. 219).

Was lernen diejenigen daraus, die anderen Menschen politische Lernprozesse mit dem Ziel einer besseren, demokratischeren und freieren Welt nahebringen wollen?

Um kein Missverständnis aufkommen zu lassen: ich sehe durchaus viele Entwicklungen durch das Netz, die Demokratie sehr gut bekommen: mehr Partizipation, mehr Transparenz, mehr Kontrolle. Aber das ist jetzt nicht mein Thema - mich beunruhigt die dunkle Seite des globalisierten Netzes.

Vor ca. 25 Jahren bereits hat Ralf Dahrendorf in den westlichen Demokratien den Verlust der „Ligaturen“ beschrieben. Das sind „tiefe kulturelle Bindungen, die Menschen in die Lage versetzen, ihren Weg durch die Welt der Optionen zu finden" (Dahrendorf 1992, S. 41). In der modernen Demokratie übernahmen diese Aufgabe beispielsweise Parteien, Kirchen und Gewerkschaften. Sie alle müssen seit Jahren einen massiven und offensichtlich unaufhaltsamen Schwund ihrer Mitglieder hinnehmen. Die Folgen für eine repräsentative Demokratie und für die Bindekräfte einer auf Humanität und sozialen Ausgleich verpflichtete Gesellschaft sind jetzt schon absehbar.

Konkret wird das durch eine aktuelle in diesem Jahr veröffentlichten repräsentative Befragung. Es geht um das „Vertrauen in Einrichtungen und Organisationen“. 13 wurden genannt, die Befragten sollten angeben, zu welchen sie Vertrauen hätten, zu welchen das „teils/teils“ der Fall wäre und zu welchen sie kein Vertrauen hätten. Auf den letzten Platz kamen die politischen Parteien mit einem Vertrauensbonus von gerade $23,1 \%$. Vor ihnen rangierten die Kirchen mi einem Vertrauenszuschuss von nur 31,1\%. Bezeichnend für den hier diskutierten Zusammenhang ist, dass der private 
Rundfunk und die sozialen Medien mit 35\% bzw. 36\% für vertrauenswürdiger eingeschätzt wurden als Kirchen und politische Parteien. Auf den ersten Platz kam übrigens die Polizei mit 65,5\%, gefolgt vom Bundesverfassungsgericht mit 63,5\% (Decker/Kies/Brähler 2016, S. 60).

In dieser Situation soll politische Bildung für politisches Engagement werben. Das ist ein schwierig zu lösender Anspruch. Aber politische Bildung darf nicht gleichgesetzt werde mit dem Handeln der etablierten politischen Akteure.

\section{Zweiter Brennpunkt: der Bildungs- und Politikbegriff}

Bei politischer Bildung geht es ... um Politik und ... um Bildung. Wer wollte das bezweifeln? Doch niemand kann definieren, was in einer an Informationen überbordenden Gesellschaft für die Millionen Einzelnen mit ihren jeweils unterschiedlichen, auch gegensätzlichen Voraussetzungen und Interessen relevante Kenntnisse und notwendiges Wissen sind, um von da zur „Bildung” zu kommen. Der Bildungsbegriff ist ein schier unendliches Thema, oft beschrieben, oft einseitig festgelegt, oft instrumentalisiert, oft missbraucht. Zweifelsohne ist Bildung „vor allem Selbstbildung” (Hastedt 2012, S. 7). Da ist der wohl einzige gemeinsame Nenner, immerhin: Bilden müssen sich die Menschen selbst.

Gibt es dabei „Lernziele”? Zumindest dieses: „Zusammenhang herstellen!“ (Negt 2010, 207). Es geht um die „Wiederherstellung der wirklichen Zusammenhänge der Welt. Der aufgeklärte Mensch ist der diese Zusammenhänge begreifende Mensch, und das ist die Grundlage seiner Mündigkeit" (Negt 2010, S. 211).

Das ist schwer in einer Gesellschaft, die als pluralisiert, individualisiert, ja als fragmentiert beschrieben wird.

In dem Zitat von Oskar Negt tauchen zwei zentrale Begriffe auf, die alles umfassen, was Bildung insgesamt, aber politische Bildung im Besonderen ausmacht, ihr zu Grunde liegen und wobei sie die Teilnehmer/-innen ihrer Veranstaltungen unterstützen soll: Aufklärung und Mündigkeit.

Die klassische Definition von Aufklärung hat Kant gegeben:

Aufklärung ist der Ausgang des Menschen aus seiner selbst verschuldeten Unmündigkeit. Unmündigkeit ist das Unvermögen, sich seines Verstandes ohne Leitung eines anderen zu bedienen. Sapere aude! Habe Mut, dich deines eigenen Verstandes zu bedienen! Ist also der Wahlspruch der Aufklärung. (Kant 1995, 162) 
Diese Aussage aus dem Jahr 1784 überragt die Zeiten.

In der Mündigkeit des Subjekts erfüllt Bildung ihren Zweck (siehe Mollenhauer 2007, S. 252). Eine wie auch immer geartete Bevormundung, Fremdbestimmung und Vorgabe von zu erreichenden Bildungs- oder Lernzielen widerspricht der Prämisse von der Mündigkeit.

Hier ergibt sich eine spannungsreiche Dialektik: Denn institutionalisierte und organisierte Bildung verfolgt eine Absicht, hat einen politisch gewollten Auftrag zu erledigen. „Sie enthält eine Antithese zur Mündigkeit ..., alle Institution ist Herrschaft" (Heydorn 1980, S. 97). Doch da bleibt die Entwicklung nicht stehen, der Bildungsanspruch entzieht sich letztendlich der Herrschaftsabsicht. Die Vermittlung von Bildung „enthält die Möglichkeit ... das Selbstverständliche zu bezweifeln“" (Heydorn 1980, S. 99).

Das heißt: Bildung ist immer befreiend und widerspenstig ... und damit auch politisch. Das müssen auch die aushalten, die Bildung organisieren, administrativ verwalten und finanzieren

Fast genauso so schwierig, wie es ist, den Bildungsbegriff zu definieren, ist es mit der eindeutigen Beschreibung dessen, was „Politik” ist. Da gibt es Traditionslinien, die miteinander nicht zu vereinbaren sind und Kategorien, die sich widersprechen. Es gibt mehrere Dimensionen und Handlungsfelder: Polity, Policy, Politics. Und schließlich ist Politik „entstaatlicht”, verstreut, globalisiert, findet national, bi- oder multinational, übernational und weltweit an unendlichen Orten und durch unzählige Akteure mit ebenso vielen Legitimationen, Intentionen und Interessen statt (siehe Hufer 2016, S. 9-13, Boeser-Schnebel u.a. 2016, S. 24-28).

Folgerichtig gibt es auch keine allgemein verbindliche, allseits akzeptierte Definition von politischer Bildung. Im besonderen Feld der politischen Erwachsenenbildung lassen sich Ziele nicht ohne weiteres so benennen, dass sie die Zustimmung aller unterschiedlichen Standpunkte, Sichtweisen und Theorien finden würden. Denn in der Vielfalt ihrer Organisationen, Träger, Institutionen und Einrichtungen spiegelt sich die Realität einer pluralen Demokratie. Dem entsprechend gibt es viele Ansichten darüber, was politische Bildung bezwecken soll. Letztendlich geht es in ihr aber immer darum, Verständnis für die Alternativlosigkeit einer sozialen Demokratie zu wecken und zu festigen, die demokratischen Regelungen und Entscheidungswege einsichtig zu machen, ein Engagement für die Einhaltung und Verteidigung der Menschenrechte zu bewirken und sich der Ablehnung von Extremismus, Totalitarismus und Diskriminierungen bewusst zu sein. Das versteht sich nicht von selbst, denn die Menschen werden nicht unbedingt als Demokraten geboren: „Demokratie ist die einzige politisch verfasste Ge- 
sellschaftsordnung, die gelernt werden muss - immer wieder, täglich und bis ins hohe Alter hinein" (Negt 2010, S. 174).

Wer sich einem emanzipatorischen Verständnis von Demokratie und politischer Bildung verbunden fühlt, für den gilt, was Oskar Negt klar formuliert hat: „Politische Bildung kann nicht gelingen, wenn die Systemfrage ausgeklammert bleibt. Wo leben wir? Was sind die bestimmenden Machtund Herrschaftsverhältnisse? Wenn diese Fragen als modernisierungsschädlich ausgegrenzt werden, ist politische Bildung lediglich Verdoppelung der Realität" (Negt 2010, S. 24).

Damit stellt sich eine wirklich brennende Frage für das pädagogische Personal.

\section{Dritter Brennpunk: die Profession}

Das demokratietheoretisch und von der Bildungsidee her zweifelsfrei gut zu begründete Postulat von Oskar Negt kollidiert oft mit der Möglichkeit, auch Taten folgen zu lassen. Eine kompromisslose Kritik der herrschenden Strukturen schafft Reibungen und Konflikte, zumal die Trägerorganisationen (Kommunen, Parteien, Kirchen, Verbände) selbst Teile dieses herrschenden Systems sind. Das zeigt sich insbesondere, wenn eine Bildungsveranstaltung handlungsorientiert angelegt ist - ein notwendiges Ziel politischer Bildung! - und sich daraus Aktivitäten entwickeln. Da kann es leicht zu Problemen und Repressionen kommen. Das Verhältnis von Reflexion und Aktion ist in der politischen Erwachsenenbildung prinzipiell spannungsgeladen (siehe Hufer 2011 und 2012).

Fritz Borinski, der Nestor der politischen Erwachsenenbildung der frühen Bundesrepublik, hatte bereits 1954 in seinem wegweisenden und immer noch lesenswerten Buch „Der Weg zum Mitbürger“ geschrieben: Den "Lehrer" der politischen Bildung solle „Mut zur Wahrheit und Freiheit" auszeichnen: „Er muß den Mut haben, gegen den Strom zu schwimmen und von der Meinung, die »man« zu vertreten hat und die »man« auch von ihm erwartet, abzuweichen" (Borinski 1954, S. 167). Das ist heute, wenn sich auf eine Stelle in der Erwachsenenbildung hunderte Universitätsabsolventen bewerben und dann oft nur ein befristeter Job herauskommt, nicht mehr selbstverständlich. Wer zu viel Mut zeigt, riskiert unter Umständen seinen Arbeitsplatz oder bekommt ihn erst gar nicht.

Ganz und gar nicht problemfrei sind auch das professionelle Verständnis und das Berufsbild politischer Erwachsenenbildner/-innen. Denn sie ar- 
beiten ja in unterschiedlichen Bezügen, Organisationen und Institutionen. Die Handlungsfelder sind - dem pluralen Verständnis von Erwachsenenbildung entsprechend - vielfältig und an zahlreichen, unterschiedlichen Orten platziert. Es ist ein Unterschied, ob jemand beispielsweise gut dotierter verbeamteter Fachbereichsleiter einer großstädtischen Volkshochschule, angestellter Bildungsreferent in einem gewerkschaftlichen Bildungszentrum oder freiberufliche Honorarkraft in gleich mehreren Institutionen und Organisationen ist. So entwickeln sich spezifische Praxiserfahrungen und -kenntnisse, die professionelles Handel generieren und manifestieren.

Und was die Profession besonders beschäftigen muss, ist die Tatsache, dass viele der nebenberuflich, oft auch hauptberuflich tätigen Honorarkräfte in prekären Verhältnissen nicht selten am Rande des Existenzminimums leben (Dobischat/Hufer 2012). In den Volkshochschulen arbeiten $87 \%$ der dort Tätigen auf Honorarbasis (Autorengruppe 2016, S. 152), nicht alle schlecht bezahlt, aber sehr viele doch.

Kann daher von einem Berufsbild/einer Profession die Rede sein? Wenn ja, was sind die Kennzeichen und Kriterien, die unbezweifelbaren und unverwechselbaren Standards der Profession?

Dieses Fragen sind zwei Projekte nachgegangen (Hufer/Richter 2014, Hufer u.a. 2013).

Herausgekommen ist einmal, dass sich die Profession der politischen Erwachsenenbildner/-innen erheblich von der der Politiklehrer/-innen an den allgemeinbildenden Schulen unterscheidet. Das geht so weit, dass sich die Frage stellt, ob es nicht zwei komplett unterschiedliche Berufe sind. Zum anderen aber kann man für die politische Erwachsenenbildung feststellen, dass es trotz ihrer großen Heterogenität doch gemeinsame professionelle Standards gibt. Es gibt Kompetenzen, die jeder braucht, um in diesem Feld professionell zu arbeiten. Christine Zeuner hat hierfür folgende Kompetenzen genannt: Fachkompetenz, Methodenkompetenz, soziale Kompetenz und reflexive Kompetenz" (Zeuner 2013, S85).

Das versteht sich nicht von selbst. Vieles geht unter im alltäglichen institutionellen Pragmatismus. Daher muss daran gearbeitet werden, dass diese Kompetenzen bewahrt, gepflegt und kollegial diskutiert werden. Dafür bedarf es Raum, Zeit, Förderung und Knowhow. 


\section{Vierter Brennpunkt: die Themen}

Es gibt gegenwärtig zwei Tendenzen, die die Existenz öffentlich geförderter politischer Bildung gefährden: Einmal wird der Staat „verschlankt“, viele Einrichtungen werden privatisiert und/oder einem „Markt“ übergeben. Aus dieser neoliberalen Grundhaltung heraus werden Einrichtungen aus den Bereichen Kultur, Soziales und Bildung zur Disposition gestellt. Zum anderen geht damit einher auch eine Umwidmung von Bildung zu Kompetenzen, entfernt man sich von den Ideen der Aufklärung und ersetzt diese durch eine immer wieder propagierte und nahezu ausschließlich geforderte Notwendigkeit zur Employability und individuellen Qualifizierung. Das sind Entwicklungen, die politischer Bildung - zumindest in der von mir beschriebenen Auffassung - diametral entgegenstehen.

Daher sei noch einmal an die Unverzichtbarkeit von politischer Bildung erinnert. Das soll in knappen Thesen geschehen:

- Demokratie lebt von Demokraten. Orte, an denen sie sich treffen, sich informieren, ihre Meinungen und Interessen austauschen und ihre politischen Ideen abgleichen, Solche Orte sind die Veranstaltungen zur politischen Bildung.

- Der modernen Gesellschaft gehen die „Ligaturen“ verloren, Davon war schon die Rede. Die Foren, die politische Bildung anbietet, können sie zwar nicht ersetzen, aber in einer zunehmend unübersichtlichen Welt Rückbesinnung, Bindungen und Orientierungen ermöglichen.

- In einer Zeit, in der sich fast alles von einem (nicht mehr vorhandenen) Zentrum weg zu diversen Orten der Peripherie verlagert, sind Plätze und Kräfte erforderlich, die das allgemein Verbindliche einfordern und zusammenhalten. Auch hier kann politische Bildung einen wesentlichen Beitrag leisten. „Die politische Bildung hat gemeinsam mit der kulturellen, der ethischen Bildung und anderen Bereichen der humanistischen Bildung für den Grundwasserstand des gesellschaftlichen Zusammenhalst zu sorgen“" („Politische Bildung ist ...“2015, 18)

- Immer mehr Bürger/-innen und Bürger zeigen Aversionen gegen die etablierte Politik. Die Institutionen der politischen Bildung können Brükken sein, die von der totalen Entfremdung wieder zurückführen. Sie können es zumindest versuchen. Die optimistische Überzeugung, dass Aufklärung durch Vernunft möglich sei, gehört zu den Grundprinzipien politischer Bildung. Bertolt Brecht lässt seinen Galiliei sagen: „Die Verführung, die von einem Beweis ausgeht, ist zu groß. Ihr erliegen die meisten, auf Dauer alle. 
Das Denken gehört zu den größten Vergnügungen der menschlichen Rasse“ (Brecht 1968, 35).

- Trash-TVs und Oberflächen-Statements in Talkshows banalisieren gesellschaftspolitische Themen. Statt einer solchen Desinformation sorgt politische Bildung für gründliche und argumentationssichere Meinungsbildung.

- In einer höchst komplexen, diffundierenden und zerstreuten Gesellschaft stellt sich die Frage nach dem Kompass und danach, welche Ethik die Richtung bestimmt. Bei den Veranstaltungen der politischen Bildung können sich Bürgerinnen und Bürger treffen, um ihre eigenen Wege und Maßstäbe in der allgemeinen Unsicherheit und Unübersichtlichkeit zu finden.

- Wenn das Leben immer mehr betriebswirtschaftlich gedacht und in fast allen Facetten nur noch so entschieden wird, wenn nur noch der eigene ökonomische Vorteil zählt, dann bleiben Gerechtigkeit und Solidarität auf der Strecke. Die „Freiheit“ wird zur Freiheit der Konsumentscheidung. So wird der moralische Kitt für eine Gesellschaft verbraucht. Dagegen ist es eine elementare Leitidee von politischer Bildung, das Wechselverhältnis von Freiheit und Gleichheit/Gerechtigkeit auszuloten.

- Die fortschreitende Globalisierung bringt es mit sich, dass sich immer mehr Menschen zunächst als Fremde begegnen. In den Veranstaltungen der politischen Bildung werden Vorurteile in Frage gestellt, Begegnungen geschaffen, einander „Fremde“ lernen sich kennen und verstehen.

- Eine zivile Gesellschaft lebt von der wechselseitigen Anerkennung unterschiedlicher Lebensentwürfe, Kulturen und Herkunft: Diese ist aber durch Fundamentalismen, autoritäre Einstellungen und totalitäre Ideologien gefährdet. Politische Bildung ist dagegen eine_Anwältin für Menschenrechte und Toleranz.

- Schließlich komme ich auf einen bereits erwähnten Punkt zurück: Das Internet hat einerseits neue Kommunikationsformen und zivilgesellschaftliche Kampagnen ermöglicht. Das ist ein Potenzial, das Demokratie nützt. Andererseits hat das weltweite Netz auch zu Solipsismus und Rückzug geführt. Das wiederum gefährdet Demokratie, die von einer vitalen Öffentlichkeit lebt. Politische Bildung ist herausgefordert, das eine zu fördern und das andere zu problematisieren. Mit Hilfe des Webs unterstützt sie politische Reflexion und stellt antipolitische Ressentiments in Frage.

Insgesamt sehe ich, dass die Zentrifugalkräfte der Gesellschaft schwinden, das, was sie zusammenhält, erodiert.

Der in Deutschland nach wie vor beliebte Schriftsteller, Kinderbuchautor und Humorist Erich Kästner hat mit wenigen Worten gezeigt, wie leicht 
es ist, etwas zu verbessern: „Es gibt nichts Gutes, außer: Man tut es“ (Kästner 1969, Bd. 1, 324). Das gilt auch in entgrenzten Zeiten. Die politische Bildung ist ein Beispiel dafür, dass „das Gute“ getan werden kann.

\section{Literatur}

Autorengruppe Bildungsberichterstattung (2016), Bildung in Deutschland 2016. Ein indikatorengestützter Bericht mit einer Analyse zu Bildung und Migration, Bielefeld.

Balzter Nadine, Ristau Yan, Schröder Achim: (2014) Wie politischer Bildung wirkt. Wirkungsstudie zur biographischen Nachhaltigkeit politischer Jugendbildung, Schwalbach/Ts.

Boeser-Schnebel Christian, Hufer, Klaus-Peter, Schnebel Karin, Wenzel, Florian (2016), Politik

wagen. Ein Argumentationstraining, Schwalbach/Ts.

Borinski Fritz (1954), Der Weg zum Mitbürger. Die politische Aufgabe der freien Erwachsenenbildung in Deutschland, Düsseldorf und Köln.

Brecht Bertolt (1968), Leben des Galiliei, 8. Aufl., Frankfurt.

Bundesministerium des Inneren (Hrsg.) (1992), Verfassungsschutzbericht 2015, Berlin.

Dahrendorf Ralf (1992), Der moderne soziale Konflikt, Stuttgart 1992.

Decker Oliver, Kiess Johannes, Brähler Elmar (Hrsg.) (2016): Die enthemmte Mitte. Autoritäre und rechtsextreme Einstellung in Deutschland, Gießen.

Dobischat Rolf, Hufer Klaus-Peter (Hrsg.) (2012), Weiterbildung im Wandel, Programm und Profil auf Profitkurs, Schwalbach/Ts.

Hastedt Heiner (Hrsg.) (2012), Was ist Bildung? Eine Textanthologie, Stuttgart.

Heydorn, Heinz-Joachim (1980), Ungleichheit für alle. Zur Neufassung des Bildungsbegriffs.

Bildungstheoretische Schriften 3, Frankfurt/M.

Hufer Klaus-Peter (2011), Politische Bildung und politische Aktion. Rekonstruktion der Kontroversen und der Konsequenzen seit den 60er Jahren, in: Benedikt Widmaier, Frank Nonnenmacher (Hrsg.), Partizipation als Bildungsziel. Politische Aktion in der politischen Bildung, Schwalbach/Ts., S. 67-81.

Hufer Klaus-Peter (2012), Reflexion oder Aktion - Die Diskussion in der politischen Erwachsenenbildung, in: Georg Weißeno/Hubertus Buchstein (Hrsg.): Politisch Handeln - Modelle, Möglichkeiten, Kompetenzen, Bonn, S. 320-334.

Hufer Klaus-Peter (2016), Politische Erwachsenenbildung. Plädoyer für eine vernachlässigte Disziplin, Bielefeld (auch erschienen als Band 1787 der Schriftenreihe der Bundeszentrale für politische Bildung, Bonn 2016). 
Hufer Klaus-Peter, Richter Dagmar (Hrsg.) (2013), Politische Bildung als Profession. Verständnisse und Forschungen, Bd. 1355 der Schriftenreihe der Bundeszentrale für politische Bildung, Bonn.

Hufer Klaus-Peter, Länge Theo, Menke Barbara, Overwien Bernd, Schudoma Laura (2013), Wissen und Können in der politischen Bildung. Wege zu professionellem Handeln, Schwalbach/Ts. 2013.

Hufer Klaus-Peter, Lange Dirk (Hrsg.) (2016), Handbuch politische Erwachsenenbildung, Schwalbach/Ts.

Kästner Erich (1969), Gesammelte Schriften für Erwachsene, Zürich 1969.

Kant Immanuel (1995), Beantwortung der Frage: Was ist Aufklärung?, in: Immanuel Kant, Werke in

sechs Bänden, Band 6, hrsg. von Rolf Toman, Köln, S. 162-170.

Mollenhauer Klaus (2007), Erziehung und Emanzipation, in: Franzjörg Baumgart (Hrsg.): Erziehungs- und Bildungstheorien. Erläuterungen - Texte - Arbeitsaufgaben, 3. Aufl., Bad Heilbrunn/Obb., S. 251-259.

Nachtwey Oliver (2016), Die Abstiegsgesellschaft. Über das Aufbegehren in der regressiven Moderne, 2. Aufl., Berlin.

Negt Oskar (2010), Der politische Mensch. Demokratie als Lebensform, Göttingen.

Politische Bildung ist die Basis des gesellschaftlichen Zusammenhalts, in: „Sächsischer Volkshochschulverband. Jahresreport 2015“, S. 16-18.

Saint Victor Jacques de (2015), Die Antipolitischen, Hamburg.

Scheidig Falk (2013), Profession, Professionalisierung und Professionalität politischer Erwachsenenbildung, in: „Journal für politische Bildung“ Nr. 4, S. 8-15.

Zeuner Christine (2013), Welchen Kompetenzbegriff sollte man in der politischen Bildung zugrunde legen?, in: Hufer Klaus-Peter, Länge Theo, Menke Barbara, Overwien Bernd, Schudoma Laura (2013), Wissen und Können in der politischen Bildung. Wege zu professionellem Handeln, Schwalbach/Ts. 2013, S. 82-86. 